\title{
Yields and Agronomic Characters of New Rice Hybrids Performed at Pine Bluff, Arkansas
}

\author{
Bihu Huang1, Zongbu Yan² \\ ${ }^{1}$ University of Arkansas at Pine Bluff, Pine Bluff, AR, USA \\ ${ }^{2}$ AgriLife Research at Beaumont, Texas A\&M University, College Station, USA \\ Email: huangb@uapb.edu
}

How to cite this paper: Huang, B.H. and Yan, Z.B. (2018) Yields and Agronomic Characters of New Rice Hybrids Performed at Pine Bluff, Arkansas. American Journal of Plant Sciences, 9, 1911-1921. https://doi.org/10.4236/ajps.2018.99138

Received: July 25, 2018

Accepted: August 20, 2018

Published: August 23, 2018

Copyright (c) 2018 by authors and Scientific Research Publishing Inc. This work is licensed under the Creative Commons Attribution International License (CC BY 4.0).

http://creativecommons.org/licenses/by/4.0/

(c) (i) Open Access

\begin{abstract}
Yields and agronomic characters of 35 new hybrids were tested at Pine Bluff, Arkansas in 2013. These hybrids were developed from 7 female parent sterile lines, UP-1s, UP-3s, 100s, 101s, 105s, 111s, and 113A, and 5 male parent restorer lines, $\mathrm{PB} 2, \mathrm{~PB} 5, \mathrm{~PB} 12, \mathrm{~PB} 13$, and PB18. The inbred variety Francis, which is a popular variety in the rice production, was used as the check (CK). Results showed that the yields of 10 hybrids were $20.3 \%-31.7 \%$ higher than that of CKFrancis. The yields of other 18 hybrids were $10.1 \%-19.4 \%$ higher than $\mathrm{CK}$, and the yields of rest of the hybrids were $2.5 \%-9.2 \%$ higher than CK. Hybrid UP-3s/PB5 had the highest yield, 12,983.8 kg/hectare and over check by $31.7 \%$. The yield of hybrid UP-3s/PB18 and 115A/PB18 were $12,333.7 \mathrm{~kg} /$ hectare and over check $25 \%$. The yields of hybrid UP-1s/PB12 was $12,324.6 \mathrm{~kg} / /$ hectare and over check $24.9 \%$. The sterile lines UP-3s, $113 \mathrm{~A}$, $105 \mathrm{~s}$, and 101s have good combinability and the average grain yields of their hybrids were over CKby 21.5\%, 20.34\%, 17.2\% and $16.2 \%$, respectively. The restorer lines $\mathrm{PB} 18, \mathrm{~PB} 5$, and $\mathrm{PB} 12$ have good combinability and the average grain yields of their hybrids were over CKby $19.9 \%, 18.4 \%$ and $16.2 \%$, respectively. These results indicated that these top hybrids and their parents of the sterile lines and restorer lines might be used in the hybrid rice breeding and production in the future.
\end{abstract}

\section{Keywords}

New Hybrid Rice, Sterile Line, Restore Line, Yields, Miller Rice and Head Rice

\section{Introduction}

Due to the increasing demand of rice with the rapidly increased in population, more and more rice have to be produced on less land and with less inputs. Hy- 
brid rice is more profitable, and more sustainable for yield production, therefore utilization of hybrid rice is an important technology to meet the increasing rice demand in world [1].

Hybrid rice is the commercial rice crop grown from F1 seeds of a cross between two genetically dissimilar parents, and have the potential of yielding 15\% $20 \%$ more than the best inbred variety grown under similar conditions [2]. Hybrids also have shown an ability to perform better under adverse conditions of drought and salinity [3]. China started hybrid rice research in 1964 and began large scale hybrid rice commercialization in 1976.

The research of hybrid rice began in USA in 1980s and released first hybrid rice in 2000 from the Rice Tec company [4]. Hybrid rice has been widely grown in the US now. It had covered about $40 \%-60 \%$ of the rice acreage in Texas during 2014 to 2016 [5] [6]. It had covered about $40 \%$ of the rice acreage in Arkansas during 2013 to 2016 [7]. Also it had covered about 25\% - 27.4\% of the rice acreage in Louisiana during 2013 to 2016 [8]. However, the price of hybrid seeds is kind of expensive currently. The farmers need more diversity and affordable hybrids in their rice production to make more profit. Therefore, hybrid rice research is very important for the objective.

Different germplasms from USDA (United States Department of Agriculture) world rice collection have been utilized and accessed in the hybrid breeding. Some new sterile lines, maintain lines, restorer lines and hybrids have been screened and bred from them. The identifying and evaluating activities have generated a lot of knowledge of hybrid breeding, selected resistant varieties and developed some hybrids from the public research program [9] [10] [11]. Hundreds new hybrids were made and identified from this research team in Arkansas. Some of those new hybrid varieties performed very well at Beaumont, Texas in past two years where is more south from Arkansas.

\section{Materials and Methods}

The research had conducted at the farm of University of Arkansas at Pine bluff, USA (UAPB) 2013. Geographical location of UAPB farm Latitude: $34^{\circ} 15^{\prime} \mathrm{N}$, Longitude: $92^{\circ} 01^{\prime} \mathrm{W}$, Elevation: 70.7 meters. Soil texture is silt loam with $\mathrm{PH}$ value of 5.3. Thirty-five hybrids were made from 7 sterile line Up-1s, UP-3s, 100 s, 101s, 105s, 111s, and 113A by crossing separately with 5 restorer lines PB2, PB5, PB12, PB13, and PB18 in 2012. The sterile line Up-1s came from the F5 generation of the Gobo (PI-369806)/Zhenshan 97//Xiangzaoxian No. 1///Jin23; 100s and 101s came from the F6 generation of the E425 (PI-442935)//Lemont/Zhenshan 97; 105s came from the F6 generation of the Gobo (PI-369806, Surinam)//Lemont/Z-97//Jin-23; 111s came from the F5 generation of the Madagascar 1283 (PI-317514)///Lemont/you-1//IR2061; 113A came from the B5F1 generation of IgnapCatelo (PI-373138)/113B; Sterile UP-3s came from Gobo (PI369806, a native rice variety of Surinam in South America)/Zhenshan 97//Xiangzaoxian No. 1, F6 generation. UP-3s was bred in University of Arkan- 
sas at Pine Bluff (UAPB). It is a two-line system sterile and carries the Dominant Early Maturity Gene. Its plant height is about $90 \mathrm{~cm}$ and heading days 85 days. The maturity dates of the progeny hybrids are depending on the maturity of female parent sterile line. Using Up-3s sterile line crossing with different restorer lines can get the earlier mature hybrids. We had developed some early maturity hybrid rice combinations by usingUP-3s crossed with male parents of different late maturity restorer lines in UAPB rice research program 2011 and 2012 [8] [10] (Huang et al. 2015 and 2016).

The restorer line PB2 was from the F5 generation of CDR210//Katy/Minghui63; PB5 was from the Katy/Minghui63//R647; PB12 was from the Lemont/Minghui63//Jasmine-85; PB13 was from Katy/Minghui63//02428; and PB18 was from Katy/Minghui63// Jasmine-85 (Table 1).

The 35 new hybrids from those sterile and restore parents and the check inbred Francis were tested for agronomic traits and yield at Pine bluff, AR in 2013. The test entries were sowed at spring 2013 in the greenhouse and transplanted to field with 20 days old seedlings (about four-leaf stage) at 6 inch per plant and $1 \mathrm{ft}$ row space.

The herbicide Command (1 pint/acre) and Permit ( $1 \mathrm{oz} / \mathrm{acre})$ applied in the pre-emergent herbicides condition on April 24.

Nitrogen fertilizer was applied $200 \mathrm{~kg} \mathrm{~N} / \mathrm{ha}$. The flood was maintained throughout the growing season. Heading dates were recorded when $50 \%$ of the plants were headed. Panicles of each plot with 1.52 meter $\times 2.43$ meter $=(3.6916$ $\mathrm{m}^{2} /$ plot) were harvested about 40 days after heading. Plant heights were measured before harvest. Yield, milled rice, and head rice also were measured.

Daily maximum temperatures (Table 2) were recorded by the sensor of NRCS Arkansas scan sites which is 50 meters away from field of study [12]. Average Yields and stand error of the mean were analyzed by SAS 9.2.

\section{Results}

1) The grain yield of new hybrid rice

a) Specific hybrids

The results showed that all the yields of hybrids are higher than check Francis (Table 3 ). The yields of top 10 hybrid rice were $20.3 \%-31.7 \%$ higher than that of check Francis; yields of middle 18 hybrids were $10.1 \%-19.4 \%$ higher than that of check Francis; and yields of last 7 hybrids were $2.6 \%-9.2 \%$ over than that of check Francis (Table 3 ).

The top 10 hybrids as follows:

The hybrid UP-3s/PB5 yielded 12,983.8 kg/hectare and 31.7\% higher than check Francis.

The hybrid UP-3s/PB18 and 113A/PB18 yielded 12,333.7 kg/hectare and 25\% higher than check Francis.

The hybrid UP-1s/PB12 yielded 12,324.6 kg/hectare and 24.9\% higher than check Francis. 
Table 1. Pedigree of the of the parents of the Thirty-five hybrids.

\begin{tabular}{|c|c|c|c|}
\hline $\begin{array}{c}\text { Name of } \\
\text { parent }\end{array}$ & Pedifree & Genaration & Note \\
\hline UP-1s & $\begin{array}{c}\text { Gobo (PI-369806)/Zhenshan 97//Xiangzaoxian } \\
\text { No. 1///Jin23 }\end{array}$ & F5 & $\begin{array}{l}\text { Sterile line } \\
\text { (female) }\end{array}$ \\
\hline UP-3s & $\begin{array}{c}\text { Gobo (PI369806)/Zhenshan 97//Xiangzaoxian } \\
\text { No. } 1\end{array}$ & F6 & $\begin{array}{l}\text { Sterile line } \\
\text { (female) }\end{array}$ \\
\hline $100 \mathrm{~s}$ & E425 (PI-442935)//Lemont/Zhenshan 97 & F6 & $\begin{array}{l}\text { Sterile line } \\
\text { (female) }\end{array}$ \\
\hline $101 \mathrm{~s}$ & E425 (PI-442935)//Lemont/Zhenshan 97 & F6 & $\begin{array}{l}\text { Sterile line } \\
\text { (female) }\end{array}$ \\
\hline $105 \mathrm{~s}$ & $\begin{array}{c}\text { Gobo (PI-369806, } \\
\text { Surinam)//Lemont/Zehnshan97//Jin-23 }\end{array}$ & F6 & $\begin{array}{l}\text { Sterile line } \\
\text { (female) }\end{array}$ \\
\hline $111 \mathrm{~s}$ & $\begin{array}{c}\text { Madagascar 1283 } \\
(\mathrm{PI}-317514) / / / \text { Lemont/you-1//IR2061 }\end{array}$ & F5 & $\begin{array}{l}\text { Sterile line } \\
\text { (female) }\end{array}$ \\
\hline $113 \mathrm{~A}$ & $\begin{array}{c}\text { IgnapCatelo } \\
(\mathrm{PI}-373138) / 113 \mathrm{~B}(\mathrm{IR} 2061-214-3-3-32 / \operatorname{Jin} 23)\end{array}$ & $\mathrm{B} 5 \mathrm{~F} 1$ & $\begin{array}{l}\text { Sterile line } \\
\text { (female) }\end{array}$ \\
\hline PB2 & CDR210//Katy/Minghui63 & F7 & $\begin{array}{l}\text { Restorer line } \\
\quad(\text { male })\end{array}$ \\
\hline PB5 & Katy/Minghui63//R647 & F7 & $\begin{array}{l}\text { Restorer line } \\
\quad(\text { male })\end{array}$ \\
\hline PB12 & Lemont/Minghui63//Jasmine-85 & F7 & $\begin{array}{l}\text { Restorer line } \\
\quad(\text { male })\end{array}$ \\
\hline PB13 & Katy/Minghui63//02428 & F7 & $\begin{array}{l}\text { Restorer line } \\
\quad(\text { male })\end{array}$ \\
\hline PB18 & Katy/Minghui63//Jasmine- 85 . & F7 & $\begin{array}{l}\text { Restorer line } \\
\quad(\text { male })\end{array}$ \\
\hline
\end{tabular}

Table 2. Daily maximum air temperature $\left({ }^{\circ} \mathrm{C}\right)$ in the UAPB field from June to October 2013.

\begin{tabular}{cccccc}
\hline Day & June & July & August & September & October \\
\hline 1 & 26.67 & 28.33 & 31.67 & 36.67 & 27.78 \\
2 & 26.11 & 28.89 & 32.78 & 33.33 & 28.89 \\
3 & 26.11 & 28.89 & 31.67 & 32.22 & 28.89 \\
4 & 28.33 & 29.44 & 32.78 & 31.11 & 28.89 \\
5 & 32.22 & 31.67 & 35.00 & 31.67 & 28.33 \\
6 & 25.00 & 31.11 & 36.11 & 33.33 & 22.22 \\
7 & 27.22 & 32.78 & 35.00 & 36.11 & 21.67 \\
8 & 28.33 & 33.33 & 36.67 & 36.11 & 22.22 \\
9 & 29.44 & 34.44 & 35.00 & 36.67 & 25.56 \\
10 & 32.78 & 36.11 & 31.11 & 35.00 & 25.56 \\
11 & 33.89 & 34.44 & 35.56 & 33.33 & 26.67 \\
12 & 33.33 & 31.11 & 34.44 & 33.33 & \\
\hline
\end{tabular}




\section{Continued}

\begin{tabular}{|c|c|c|c|c|c|}
\hline 13 & 34.44 & 29.44 & 28.89 & 30.00 & \\
\hline 14 & 31.11 & 30.56 & 26.11 & 25.56 & \\
\hline 15 & 31.11 & 32.22 & 25.56 & 30.56 & \\
\hline 16 & 31.11 & 31.67 & 24.44 & 32.22 & \\
\hline 17 & 28.89 & 33.89 & 27.22 & 32.78 & \\
\hline 18 & 27.78 & 33.33 & 27.22 & 33.33 & \\
\hline 19 & 31.67 & 32.78 & 30.00 & 32.78 & \\
\hline 20 & 32.78 & 33.89 & 31.67 & 26.67 & 24.44 \\
\hline 21 & 32.78 & 33.33 & 32.78 & 24.44 & 22.22 \\
\hline 22 & 33.33 & 33.89 & 33.33 & 24.44 & 19.44 \\
\hline 23 & 33.33 & 32.22 & 33.33 & 26.67 & \\
\hline 24 & 32.78 & 30.56 & 33.33 & 30.56 & \\
\hline 25 & 32.22 & 29.44 & 32.22 & 27.78 & \\
\hline 26 & 33.33 & 26.11 & 32.22 & 28.89 & \\
\hline 27 & 35.56 & 28.33 & 32.78 & 29.44 & \\
\hline 28 & 35.00 & 30.56 & 33.33 & 30.00 & \\
\hline 29 & 32.22 & 31.67 & 34.44 & 22.78 & \\
\hline 30 & 28.33 & 31.67 & 35.00 & 27.22 & \\
\hline 31 & & 31.67 & 36.67 & & \\
\hline Average & 30.91 & 31.54 & 32.20 & 30.83 & 25.20 \\
\hline
\end{tabular}

Table 3. The grain yield of new hybrids at Pine Bluff, Arkansas 2013.

\begin{tabular}{|c|c|c|c|c|c|c|c|}
\hline \multirow{2}{*}{ Order } & \multirow{2}{*}{$\begin{array}{c}\text { Cross } \\
\text { Sterile/Restore }\end{array}$} & \multicolumn{2}{|c|}{ Average WT per plot } & \multicolumn{2}{|c|}{ Average yield per hectare } & \multirow{2}{*}{$\begin{array}{c}\text { Over CK } \\
\quad(\%)\end{array}$} & \multirow{2}{*}{$\begin{array}{c}\text { Rank of } \\
\text { the } 36 \\
\text { entries }\end{array}$} \\
\hline & & $\mathrm{Kg} /$ Plot & Std Err & $\mathrm{Kg} /$ Hectare & Std Err & & \\
\hline 9 & UP-3s/PB5 & 4.79 & 0.15 & $12,983.8$ & 402.21 & 31.7 & 1 \\
\hline 30 & UP-3s/PB18 & 4.55 & 0.03 & $12,333.7$ & 72.25 & 25.0 & 2 \\
\hline 35 & $113 \mathrm{~A} / \mathrm{PB} 18$ & 4.55 & 0.04 & $12,333.6$ & 95.51 & 25.0 & 2 \\
\hline 15 & UP-1s/PB12 & 4.55 & 0.06 & $12,324.6$ & 149.35 & 24.9 & 3 \\
\hline 33 & 105s/PB18 & 4.54 & 0.02 & $12,297.5$ & 62.58 & 24.6 & 4 \\
\hline 14 & 113A/PB5 & 4.53 & 0.04 & $12,261.4$ & 95.59 & 24.2 & 5 \\
\hline 32 & 101s/PB18 & 4.52 & 0.70 & $12,261.4$ & 186.56 & 24.2 & 5 \\
\hline 18 & 101s/PB12 & 4.39 & 0.06 & $11,900.3$ & 164.37 & 20.6 & 6 \\
\hline 12 & 105s/PB5 & 4.38 & 0.13 & $11,873.2$ & 349.83 & 20.3 & 7 \\
\hline 28 & $113 \mathrm{~A} / \mathrm{PB} 13$ & 4.38 & 0.12 & $11,873.1$ & 334.08 & 20.3 & 7 \\
\hline 7 & $\mathrm{Ji3A} / \mathrm{PB} 2$ & 4.35 & 0.14 & $11,782.8$ & 383.97 & 19.4 & 8 \\
\hline 26 & $105 \mathrm{~s} / \mathrm{PB} 13$ & 4.35 & 0.08 & $11,773.8$ & 221.86 & 19.3 & 9 \\
\hline 23 & UP-3s/PB13 & 4.33 & 0.08 & $11,746.7$ & 227.84 & 19.1 & 10 \\
\hline
\end{tabular}




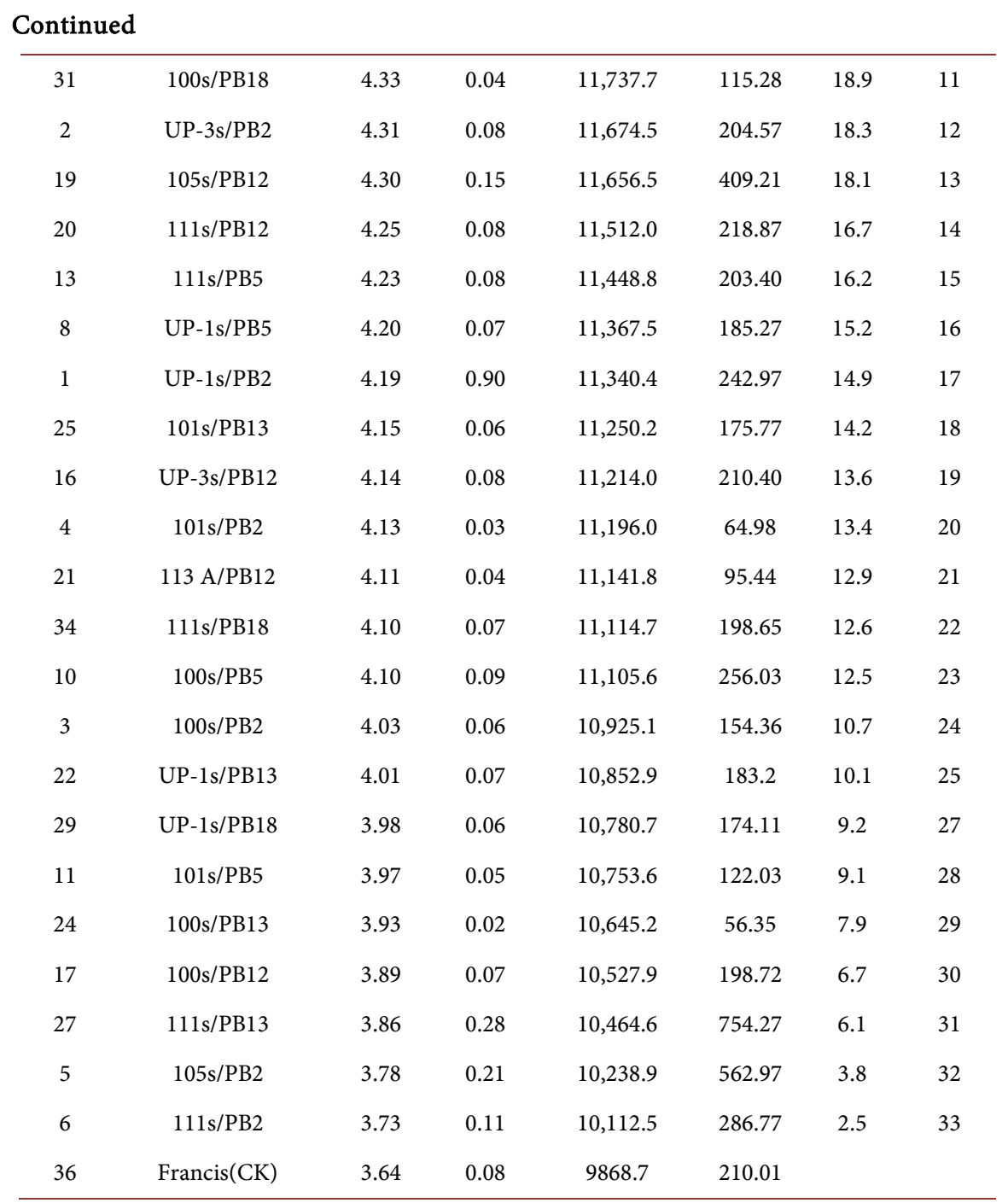

The hybrid 105s/PB18 got grain yield 12,297.5 kg/hectare and $24.6 \%$ higher than check Francis.

The hybrid 113A/PB5 and 101s/PB18 yielded 12,261.4 kg/hectare and 24.2\% higher than check Francis.

The hybrid 101s/PB12 yielded 11,900.3 kg/hectare and 20.6\% higher than check Francis.

The hybrid 105s/PB5 and 113A/PB13 yielded 11,873.2 kg/hectare and 20.3\% higher than check Francis.

b) Average yields of same sterile lines crossing 6 restorer lines and a same restorer line crossing 6 sterile lines.

Table 4 (horizontally) listed the average yields of hybrids from the same sterile line crossed with 5 different restorer lines, PB2, PB5, PB12, PB13 andPB18:

i) Hybrids developed from sterile line UP-3s had the highest average yield of $11,990.5 \mathrm{~kg} / \mathrm{hectare}$ and over CK by $21.5 \%$.

ii) Hybrids developed from sterile line $113 \mathrm{~A}$ had the second high average yield of $11,878.5 \mathrm{~kg} / \mathrm{hectare}$ and over CK by $20.34 \%$. 
Table 4. Average hybrid yields from one parent line crossing other relevant and different parent lines.

\begin{tabular}{|c|c|c|c|c|c|c|c|}
\hline $\begin{array}{l}\text { Restore } \\
\text { lines } \\
\text { Sterile } \\
\text { lines }\end{array}$ & PB2 & PB5 & PB12 & PB13 & PB18 & Average & $\begin{array}{c}\text { Over } \\
\text { CK (\%) }\end{array}$ \\
\hline \multicolumn{8}{|c|}{ Yield Kg/Hectare } \\
\hline UP-1s & $11,340.4$ & $11,367.5$ & $12,324.6$ & $10,852.9$ & $10,780.7$ & $11,333.2$ & 14.80 \\
\hline UP-3s & $11,674.5$ & $12,983.8$ & $11,214.0$ & $11,746.7$ & $12,333.7$ & $11,990.5$ & 21.50 \\
\hline $100 \mathrm{~s}$ & $10,925.1$ & $11,105.6$ & $10,527.9$ & $10,645.2$ & $11,737.7$ & $10,988.3$ & 11.30 \\
\hline $101 \mathrm{~s}$ & $11,196.0$ & $10,753.6$ & $11,900.3$ & $11,250.2$ & $12,261.4$ & $11,472.3$ & 16.20 \\
\hline $105 \mathrm{~s}$ & $10,238.9$ & $11,873.2$ & $11,656.5$ & $11,773.8$ & $12,297.5$ & $11,568.0$ & 17.20 \\
\hline $111 \mathrm{~s}$ & $10,112.5$ & $11,448.8$ & $11,512.0$ & $10,464.6$ & $11,114.7$ & $10,930.5$ & 10.80 \\
\hline $113 \mathrm{~A}$ & $11,782.8$ & $12,261.4$ & $11,141.8$ & $11,873.1$ & $12,333.6$ & $11,878.5$ & 20.34 \\
\hline Average & $11,038.6$ & $11,684.8$ & $11,468.2$ & $11,229.5$ & $11,837.0$ & $11,451.6$ & 16.00 \\
\hline Over CK (\%) & 11.9 & 18.4 & 16.2 & 13.8 & 19.9 & 16 & \\
\hline
\end{tabular}

iii) Hybrids developed from sterile line 105s had the third high average yield of $11,568.0 \mathrm{~kg} /$ hectare and over CK $17.2 \%$.

iv) Hybrids developed from sterile line 101s had the fourth high average yield of $11,472.3 \mathrm{~kg} /$ hectare and over CK Francis $16.2 \%$.

v) Hybrids developed from sterile line UP-1s had the average yield of 11,333.2 $\mathrm{kg} /$ hectare and over CK $14.8 \%$.

vi) Hybrids developed from sterile line 100s had the average yield of 10,988.3 $\mathrm{kg} / \mathrm{hectare}$ and over CK $11.3 \%$.

vii) Hybrids developed from sterile line 111s had the lowest average yield of 10,930.5 kg/hectare and but still over CK Francis 10.8\%.

Table 4 (vertically) listed the average yields of hybrids for the same restorer line crossed with 6 different sterile lines UP-1s, UP-3s, 100s, 101A, 105s, 101s and 113A.

i) Hybrids from PB18 crossed with the 7 sterile lines had the highest average yield $11,837.0 \mathrm{~kg} /$ hectare and was over CK Francis $19.9 \%$.

ii) Hybrids from PB5 crossed with the 7 sterile lines had the second high average yield of $11,684.8 \mathrm{~kg} /$ hectare and was over CK Francis $18.4 \%$.

iii) Hybrids from PB12 crossed with the 7 sterile lines had the third high average yield of $11,468.2 \mathrm{~kg} /$ hectare and was over CK Francis $16.2 \%$.

iv) Hybrids from PB13 crossed with the 7 sterile lines had the fourth high average yield of 11,229.5 kg/hectare and was over CKFrancis $13.8 \%$.

v) Hybrids from PB2 crossed the 7 sterile lines had the average yield of $11,038.6 \mathrm{~kg} /$ hectare and was over CK Francis $11.9 \%$.

2) The heading days from planting to heading

All the heading days of the hybrids from planting to heading were longer than CK Francis, excepting hybrid UP-3s/ PB13 was the same 75.7 days as CK Francis (Table 5). The heading days of the rest 34 hybrids were 76 days - 94.3 days 
(Table 3). Eight hybrids UP-3s/PB2, UP-3s/PB5, UP-3s/PB12, 100s/PB12, UP-3s/PB13, 100s/PB13, UP-1s/PB18, and UP-3s/PB18 were 76.0 days - 79.0 days; 3 hybrids 113A/PB12, 113A/PB13 and 113A/PB18 were 90.7 days - 94.3 days; and other 24 hybrids were 80.3 days - 87.7 days (Table 5).

3) The Plant heights

Plant heights of 35 new hybrids were $103 \mathrm{~cm}-128 \mathrm{~cm}$ (Table 5). Plant heights of the most two-line system hybrids were below $110 \mathrm{~cm}$ and the most three-line system hybrids were over $120 \mathrm{~cm}$ and lodged. The plant heights of 19 hybrids were below $110 \mathrm{~cm}$. The plant heights of 12 hybrids were $110 \mathrm{~cm}-120 \mathrm{~cm}$. The plant heights of 4 hybrids were $120.3 \mathrm{~cm}-128 \mathrm{~cm}$ (Table 5).

4) The total milling rice and head rice rates of new hybrids

The milling rice rates of 35 new hybrids were $65 \%-68.3 .7 \%$ and all were lower than Check Francis (69.1\%). However, most of the hybrids have higher total milling rice than $\mathrm{CK}$ because their total grain rice were much higher than $\mathrm{CK}$ (Table 6).

Head rice rates of 35 new hybrids were $44.9 \%$ - 59.4\%. Four hybrids 111 s/PB2, 105s/PB12, 111s/PB13, and 101s/PB18 had good head rice and their rates were $59.1 \%, 59.4 \%, 58.8 \%$, and $58.8 \%$ respectively. But all head rice rates of hybrids were lower than check Francis (63.2\%). Nevertheless, some of the hybrids had higher total head rice than CK (Table 6).

\section{Discussions}

1) Hybrids from the four sterile lines UP-3s, UP-1s, 105s and 113A, and the 3 restorer lines $\mathrm{PB} 18, \mathrm{~PB} 12$, and $\mathrm{PB} 5$ were performed well in this experiment. As parents, these four sterile lines and 3 restorer lines worth to pay more attention in the future breeding and the seed production for obtaining the high yielding hybrids. UP-1s, Up-s and 105s are two-line system sterile lines. $113 \mathrm{~A}$ is three-system sterile line. Up-3s has a dominant early maturity gene. The early maturity hybrid rice will be obtained when this sterile line crosses to late restorer lines. Up-1s is an aromatic sterile line and can use it to make high yield and aromatic hybrid rice by crossing with some restorer lines.

$\mathrm{Up}-1 \mathrm{~s}$ and Up-3s are lower amylose content sterile lines and can use them to make lower amylose content $(16 \%$ - 18\%) hybrid rice.

$105 \mathrm{~s}$ and $113 \mathrm{~A}$ are high amylose content sterile line and can use them to make higher amylose content $(20 \%-22 \%)$ hybrid rice.

2) The 7hybrids of UP-3s/ PB13, UP-3s/PB2, UP-3s/PB5, UP-3s/PB12, 100s/PB12, 100s/PB13, and 101s/PB18 were earlier heading Hybrids. The growing period of these hybrids might suit to the rice production in the south states.

3) Plant heights of most two-line system hybrids $(100-110 \mathrm{~cm})$ were lower than that of most three-line hybrids $(110-120 \mathrm{~cm})$ in this yield test. Two-line hybrids could be used in the normal or high nitrogen field condition and three-line hybrids could be used in the low nitrogen fertilizer condition of the rice production. 
Table 5. Heading dates and plant heights of new hybrids at Pine Bluff, AR 2013.

\begin{tabular}{|c|c|c|c|c|c|}
\hline \multirow{2}{*}{ Order } & \multirow{2}{*}{$\begin{array}{c}\text { Cross } \\
\text { Sterile/Restore }\end{array}$} & \multicolumn{2}{|c|}{$\begin{array}{l}\text { Days from planting to } \\
\text { heading }\end{array}$} & \multicolumn{2}{|c|}{ Plant height $(\mathrm{cm})$} \\
\hline & & Average & Std Error & Average & Std Error \\
\hline 1 & UP-1s/PB2 & 85.60 & 0.88 & 119.0 & 2.52 \\
\hline 2 & UP-3s/PB2 & 79.00 & 0.58 & 103.3 & 0.88 \\
\hline 3 & $100 \mathrm{~s} / \mathrm{PB} 2$ & 81.00 & 0.58 & 105.7 & 2.33 \\
\hline 4 & 101s/PB2 & 86.00 & 0.58 & 108.7 & 3.28 \\
\hline 5 & $105 \mathrm{~s} / \mathrm{PB} 2$ & 84.30 & 0.88 & 105.3 & 0.67 \\
\hline 6 & $111 \mathrm{~s} / \mathrm{PB} 2$ & 82.00 & 1.15 & 108.7 & 2.85 \\
\hline 7 & $113 \mathrm{~A} / \mathrm{PB} 2$ & 87.70 & 0.67 & 128.0 & 2.02 \\
\hline 8 & UP-1s/PB5 & 86.30 & 0.88 & 113.7 & 0.88 \\
\hline 9 & UP-3s/PB5 & 77.30 & 1.20 & 110.0 & 1.15 \\
\hline 10 & 100s/PB5 & 81.30 & 1.45 & 103.0 & 1.00 \\
\hline 11 & 101s/PB5 & 81.30 & 0.88 & 114.0 & 2.08 \\
\hline 12 & 105s/PB5 & 81.70 & 1.20 & 108.7 & 3.28 \\
\hline 13 & 111s/PB5 & 80.30 & 0.33 & 104.0 & 1.00 \\
\hline 14 & $113 \mathrm{~A} / \mathrm{PB} 5$ & 87.70 & 0.67 & 120.3 & 2.03 \\
\hline 15 & UP-1s/12 & 82.00 & 1.15 & 123.0 & 1.15 \\
\hline 16 & UP-3s/12 & 76.30 & 1.45 & 107.7 & 1.86 \\
\hline 17 & $100 \mathrm{~s} / 12$ & 76.30 & 1.20 & 106.0 & 4.58 \\
\hline 18 & $101 \mathrm{~s} / 12$ & 82.70 & 1.20 & 116.3 & 3.18 \\
\hline 19 & $105 \mathrm{~s} / 12$ & 81.00 & 0.58 & 110.0 & 6.51 \\
\hline 20 & $111 \mathrm{~s} / 12$ & 80.30 & 0.33 & 110.7 & 1.20 \\
\hline 21 & $113 \mathrm{~A} / \mathrm{PB} 12$ & 94.30 & 1.15 & 117.3 & 0.88 \\
\hline 22 & UP-1s/PB13 & 85.50 & 0.33 & 112.3 & 5.24 \\
\hline 23 & UP-3s/PB13 & 75.70 & 1.76 & 105.7 & 0.67 \\
\hline 24 & $100 \mathrm{~s} / \mathrm{PB} 13$ & 76.00 & 1.73 & 103.3 & 0.88 \\
\hline 25 & $101 \mathrm{~s} / \mathrm{PB} 13$ & 81.00 & 0.00 & 106.7 & 1.67 \\
\hline 26 & $105 \mathrm{~s} / \mathrm{PB} 13$ & 80.70 & 0.33 & 110.3 & 0.67 \\
\hline 27 & $111 \mathrm{~s} / \mathrm{PB} 13$ & 82.00 & 1.15 & 101.0 & 0.58 \\
\hline 28 & $113 \mathrm{~A} / \mathrm{PB} 13$ & 90.70 & 0.88 & 119.3 & 0.88 \\
\hline 29 & UP-1s/PB18 & 76.30 & 0.88 & 118.7 & 2.03 \\
\hline 30 & UP-3s/PB18 & 78.30 & 0.33 & 106.3 & 1.20 \\
\hline 31 & $100 \mathrm{~s} / \mathrm{PB} 18$ & 83.30 & 1.20 & 106.0 & 1.53 \\
\hline 32 & $101 \mathrm{~s} / \mathrm{PB} 18$ & 86.70 & 0.33 & 109.0 & 4.04 \\
\hline 33 & $105 \mathrm{~s} / \mathrm{PB} 18$ & 83.00 & 1.85 & 108.7 & 1.86 \\
\hline 34 & $111 \mathrm{~s} / \mathrm{PB} 18$ & 83.30 & 0.58 & 108.3 & 0.88 \\
\hline 35 & $113 \mathrm{~A} / \mathrm{PB} 18$ & 93.70 & 0.88 & 127.3 & 0.88 \\
\hline 36 & Francis (CK) & 75.70 & 0.67 & 105.3 & 0.33 \\
\hline
\end{tabular}


Table 6. Sample milling rice, sample head rice rates and head rice yields of the new hybrids.

\begin{tabular}{|c|c|c|c|c|c|c|c|c|c|c|c|}
\hline \multirow{2}{*}{ Order } & \multirow{2}{*}{$\begin{array}{c}\text { Cross } \\
\text { Sterile/Restore }\end{array}$} & \multicolumn{2}{|c|}{ Sample milling Rice } & \multicolumn{2}{|c|}{ Sample Head rice } & \multicolumn{3}{|c|}{ Total Milling rice } & \multicolumn{3}{|c|}{ Total head rice } \\
\hline & & $\%$ & Std Err & $\%$ & Std Err & $\mathrm{kg} / \mathrm{ha}$ & Over CK (\%) & Rank & $\mathrm{kg} / \mathrm{ha}$ & Over CK (\%) & Rank \\
\hline 1 & UP-1s/PB2 & 67.8 & 0.17 & 55.1 & 0.84 & 7688.8 & 12.8 & & 6248.6 & 0.2 & \\
\hline 2 & UP-3s/PB2 & 66.7 & 0.45 & 44.9 & 2.02 & 7786.9 & 14.1 & & 5241.9 & -16.0 & \\
\hline 3 & $100 \mathrm{~s} / \mathrm{PB} 2$ & 68.6 & 0.35 & 55.4 & 0.36 & 7494.6 & 9.9 & & 6052.5 & -3.0 & \\
\hline 4 & 101s/PB2 & 65.0 & 0.35 & 56.3 & 0.57 & 7277.4 & 6.7 & & 6303.3 & 1.1 & \\
\hline 5 & $105 \mathrm{~s} / \mathrm{PB} 2$ & 67.9 & 0.32 & 55.8 & 1.60 & 6952.2 & 1.9 & & 5713.3 & -8.4 & \\
\hline 6 & $111 \mathrm{~s} / \mathrm{PB} 2$ & 67.4 & 0.36 & 59.1 & 0.36 & 6815.8 & -0.1 & & 5976.5 & -4.2 & \\
\hline 7 & $113 \mathrm{~A} / \mathrm{PB} 2$ & 65.2 & 0.85 & 56.2 & 0.58 & 7682.4 & 12.7 & & 6621.9 & 6.2 & \\
\hline 8 & UP-1s/PB5 & 66.5 & 0.67 & 55.5 & 1.13 & 7559.4 & 10.9 & & 6309.0 & 1.2 & \\
\hline 9 & UP-3s/PB5 & 67.1 & 0.38 & 53.1 & 0.82 & 8712.1 & 27.8 & 1 & 6894.4 & 10.5 & 6 \\
\hline 10 & 100s/PB5 & 66.1 & 0.49 & 53.4 & 0.75 & 7340.8 & 7.6 & & 5930.4 & -4.9 & \\
\hline 11 & 101s/PB5 & 66.4 & 0.78 & 57.0 & 1.00 & 7140.4 & 4.7 & & 6129.6 & -1.7 & \\
\hline 12 & 105s/PB5 & 66.3 & 0.61 & 54.7 & 0.32 & 7871.9 & 15.4 & 12 & 6494.6 & 4.1 & \\
\hline 13 & 111s/PB5 & 66.1 & 0.47 & 56.7 & 0.59 & 7567.7 & 11.0 & & 6491.5 & 4.1 & \\
\hline 14 & 113A/PB5 & 66.9 & 0.33 & 56.5 & 0.38 & 8202.9 & 20.3 & 6 & 6927.7 & 11.1 & 4 \\
\hline 15 & UP-1s/PB12 & 67.6 & 0.33 & 57.7 & 0.92 & 8331.4 & 22.2 & 2 & 7111.3 & 14.0 & 2 \\
\hline 16 & UP-3s/PB12 & 67.7 & 0.35 & 50.3 & 3.18 & 7591.9 & 11.3 & & 5640.6 & -9.6 & \\
\hline 17 & $100 \mathrm{~s} / \mathrm{PB} 12$ & 68.3 & 0.36 & 57.4 & 0.22 & 7190.6 & 5.4 & & 6043.0 & -3.1 & \\
\hline 18 & 101s/PB12 & 66.9 & 0.74 & 56.7 & 1.36 & 7961.3 & 16.7 & 11 & 6747.5 & 8.2 & \\
\hline 19 & $105 \mathrm{~s} / \mathrm{PB} 12$ & 67.0 & 0.24 & 59.4 & 0.32 & 7809.5 & 14.5 & & 6924.0 & 11.0 & 5 \\
\hline 20 & 111s/BP12 & 66.7 & 0.75 & 54.6 & 3.97 & 7678.5 & 12.6 & & 6285.6 & 0.8 & \\
\hline 21 & $113 \mathrm{~A} / \mathrm{PB} 12$ & 67.4 & 0.49 & 52.5 & 0.69 & 7509.6 & 10.1 & & 5849.5 & -6.2 & \\
\hline 22 & UP-1s/PB13 & 67.6 & 0.61 & 51.9 & 1.46 & 7336.6 & 7.6 & & 5632.7 & -9.7 & \\
\hline 23 & UP-3s/PB13 & 68.2 & 0.52 & 55.4 & 0.71 & 8011.2 & 17.4 & 8 & 6507.7 & 4.3 & \\
\hline 24 & 100 s/PB13 & 67.2 & 0.49 & 54.8 & 1.02 & 7153.6 & 4.9 & & 5833.6 & -6.5 & \\
\hline 25 & 101s/PB13 & 66.9 & 0.35 & 57.3 & 0.27 & 7526.4 & 10.4 & & 6446.4 & 3.4 & \\
\hline 26 & $105 s / \mathrm{PB} 13$ & 67.9 & 0.20 & 54.6 & 1.17 & 7994.4 & 17.2 & 9 & 6428.5 & 3.1 & \\
\hline 27 & $111 \mathrm{~s} / \mathrm{PB} 13$ & 67.2 & 0.35 & 58.8 & 0.42 & 7032.2 & 3.1 & & 6153.2 & -1.3 & \\
\hline 28 & $113 \mathrm{~A} / \mathrm{PB} 13$ & 67.2 & 0.40 & 55.6 & 0.35 & 7978.7 & 17.0 & & 6601.4 & 5.8 & \\
\hline 29 & UP-1s/PB18 & 67.2 & 0.52 & 53.8 & 1.69 & 7244.6 & 6.2 & & 5800.0 & -7.0 & \\
\hline 30 & UP-3s/PB18 & 67.3 & 0.33 & 56.2 & 0.99 & 8300.6 & 21.7 & 3 & 6931.5 & 11.1 & 3 \\
\hline 31 & 100 s/PB18 & 68.0 & 0.40 & 54.9 & 0.87 & 7981.6 & 17.0 & 10 & 6444.0 & 3.3 & \\
\hline 32 & 101s/PB18 & 66.8 & 0.54 & 58.8 & 2.47 & 8190.6 & 20.1 & 5 & 7209.7 & 15.6 & 1 \\
\hline 33 & $105 \mathrm{~s} / \mathrm{PB} 18$ & 66.9 & 0.60 & 54.5 & 0.74 & 8227.1 & 20.6 & 4 & 6702.1 & 7.5 & \\
\hline 34 & 111s/PB18 & 65.4 & 1.0. & 54.4 & 1.29 & 7269.0 & 6.6 & & 6046.4 & -3.1 & \\
\hline 35 & $113 \mathrm{~A} / \mathrm{PB} 18$ & 65.6 & 0.64 & 53.8 & 2.87 & 8090.8 & 18.6 & 7 & 6635.5 & 6.4 & \\
\hline 36 & Francis (CK) & 69.1 & 0.4 & 63.2 & 0.79 & 6819.3 & I & & 6237.0 & I & \\
\hline
\end{tabular}


4) Milled head rice rate is a key determinant of rice grain quality and an important factor affecting its market value. Most hybrid rice in this test have lower head rice rate. However, 8 hybrids 111s/PB2, UP-1s/PB12, 100s/PB12, 101s/PB12, 105s/PB12, 101s/PB13, 111s/PB13, and 105s/PB18 have good head rice rates from $57.4 \%-59.4 \%$. The parents of these hybrids are developed from 5 sterile lines of UP-1s, 100s, 101s, 105s, 111s, and 4 restorer lines of PB2, PB12, PB13, and $\mathrm{PB} 18$. They are good donors in hybrid rice breeding for quality milled rice.

\section{Conflicts of Interest}

The authors declare no conflicts of interest regarding the publication of this paper.

\section{References}

[1] Virmani, S.S., Sun, Z.X., Mou, T.M., Jauhar, A. and Mao, C.X. (2003) Two-Line Hybrid Rice Breeding Manual. IRRI, Los Banos.

http://www.knowledgebank.irri.org/ricebreedingcourse/documents/2LineHybridRic eBreeding

[2] Virmani, S.S., Viraktamath, B.C., Casal, C.L., Toledo, R.S., Lopez, M.T. and Manalo, J.O. (1997) Hybrid Rice Breeding Manual . IRRI, Los Banos. Laguna, Philippines. https://www.cabdirect.org/?target=\%2fcabdirect $\% 2$ fabstract $\% 2$ f20006781640

[3] Zayed, B.A., El-Namaky, R.A., El-Refaee Y.Z. and Seedek, S.E.M. (2012) Comparative Study on Hybrid and Inbred Rice under Drought and Saline Stresses. Plant Production, 3, 91-108.

[4] Bennett, D. (2010) Hybrid Rice Varieties-Range of Options. Delta Farm Press, Saint Charles.

[5] Wilson, L.T., Yang, Y., Wang, J. and Morace, B. (2016) Texas Rice Crop Survey. https://beaumont.tamu.edu/CropSurvey

[6] Wilson, L.T. (2017) How Times Have Changed. Rice Farming. One Grower Publishing, LLC. Olive Branch, 18-19.

[7] Hardke, J. (2016) Reviewing the 2016 Arkansas rice Season. Arkansas Row Crops. University of Arkansas, Fayettivile, AR.

http://www.arkansas-crops.com/2016/11/09/reviewing-the-2016-arkansas-rice-seas on/

[8] Harrell, D. (2016) Louisiana Rice Acreage by Variety Survey Hybrid, LSU Ag-Center 101. Efferson Hall, Baton Rouge, LA.

http://www.lsuagcenter.com/topics/crops/rice/statistics/rice-varieties

[9] Huang, B. and Yan, Z. (2015) Utilizing Dominant Early Maturity Genes of Sterile Line UP-3s in Hybrid Rice Breeding to Avoid High Temperature Season. American Journal of Plant Sciences, 6, 2596-2602. https://doi.org/10.4236/ajps.2015.616262

[10] Huang, B.H. and Yan, Z.B. (2016) Performance of 32 Hybrid Rice Varieties at Pine Bluff of Arkansas. American Journal of Plant Sciences, 7, 2239-2247. https://doi.org/10.4236/ajps.2016.715197

[11] Huang, B. and Yan, Z. (2016) Straighthead and Agronomy Characters Testing for Selected Parent Lines and Hybrid Combinations at UAPB in 2011-2012. American Journal of Plant Sciences, 7, 1423-1428. https://doi.org/10.4236/ajps.2016.710136

[12] NRCS Arkansas Scan Site UAPB. http://wcc.sc.egov.usda.gov/nwcc/site? sitenum=2085 